Department of Applied Mathematics

Faculty of EEMCS

(s)

University of Twente

The Netherlands
P.O. Box 217

7500 AE Enschede

The Netherlands

Phone: +31-53-4893400

Fax: $+31-53-4893114$

Email: memo@math.utwente.nl

www.math.utwente. $\mathrm{nl} /$ publications

Memorandum No. 1709

An improved local search algorithm for 3-SAT

T. Brueggemann And W. Kern

January, 2004

ISSN 0169-2690 


\title{
An improved local search algorithm for 3-SAT
}

\author{
Tobias Brueggemann ${ }^{1}$, Walter Kern * \\ Department of Applied Mathematics, University of Twente, \\ P.O. Box 217, 7500 AE Enschede, The Netherlands
}

\begin{abstract}
We slightly improve the pruning technique presented in Dantsin et. al. (2002) to obtain an $\mathcal{O}^{*}\left(1.473^{n}\right)$ algorithm for 3-SAT.
\end{abstract}

Key words: exact algorithm, local search, 3-SAT

MSC2000: 68Q25

\section{Introduction}

An instance of 3 -SAT is a boolean formula $\varphi$ in $n$ variables $x_{1}, \ldots, x_{n}$, defined as the conjunction of a set $\mathcal{C}$ of disjunctive clauses of length at most 3 . Satisfiability of $\varphi$ can be tested in a straightforward manner in time

$$
\mathcal{O}\left(2^{n} \cdot n^{3}\right)=\mathcal{O}^{*}\left(2^{n}\right)
$$

Here, as usual, we use the $\mathcal{O}^{*}$-notation to indicate that polynomial factors are suppressed.

During the last years so-called exact algorithms have been designed solving 3-SAT in time $\mathcal{O}^{*}\left(\alpha^{n}\right)$ with $\alpha<2$, see Schoening [3] for an overview. The currently fastest randomized algorithms run in time $\mathcal{O}^{*}\left(1.3302^{n}\right)$ (see Hofmeister, Schoening, Schuler and Watanabe [2]) and the fastest deterministic algorithm (see Dantsin et. al. [1]) takes $\mathcal{O}^{*}\left(1.481^{n}\right)$. We slightly improve the pruning technique used in Dantsin et. al. [1] to obtain a running time of $\mathcal{O}^{*}\left(1.473^{n}\right)$.

\footnotetext{
* Corresponding author.

Email addresses: t. brueggemann@math. utwente.nl (Tobias Brueggemann), kern@math. utwente.nl (Walter Kern).

1 supported by the Netherlands Organization for Scientific Research (NWO) grant 613.000.225 (Local Search with Exponential Neighborhoods)
} 


\section{Local search}

Let $\varphi$ be an instance of 3-SAT given by a set $\mathcal{C}$ of clauses in variables $x_{1}, \ldots, x_{n}$. For $a \in\{0,1\}^{n}$ let $B_{r}(a) \subseteq\{0,1\}^{n}$ denote the set of 0 - 1 vectors with Hamming distance at most $r$ from $a$. The currently fastest algorithms for 3-SAT are based on local search: First, a covering code of suitable radius $r \leq n$ is constructed, i.e. a set $A \subseteq\{0,1\}^{n}$ such that

$$
\{0,1\}^{n}=\bigcup_{a \in A} B_{r}(a)
$$

holds. Next we search for a truth assignment for $\varphi$ in each $B_{r}(a), a \in A$, separately. To make our paper self-contained, we briefly describe the basic idea for constructing a covering code and (to some extent) the local search within a given $B_{r}(a)$ as presented in Dantsin et. al. [1].

Covering codes. As $B_{r}:=B_{r}(0)$ contains exactly

$$
V(n, r)=\sum_{i=1}^{r}\left(\begin{array}{l}
n \\
i
\end{array}\right)
$$

elements, a covering code $A \subseteq\{0,1\}^{n}$ of radius $r \leq n$ must necessarily satisfy

$$
|A| \geq \frac{2^{n}}{V(n, r)}
$$

Covering codes of approximately this size indeed exist and can be constructed randomly: Choose

$$
t=\frac{n 2^{n}}{V(n, r)}
$$

elements from $\{0,1\}^{n}$ uniformly at random, resulting in a set $A \subseteq\{0,1\}^{n}$ of size $|A| \leq t$. The probability that a particular $a^{*} \in\{0,1\}^{n}$ is not covered by any $B_{r}(a), a \in A$ is at most

$$
P\left[a^{*} \text { not covered }\right]=\left(1-\frac{V(n, r)}{2^{n}}\right)^{t} \leq \mathrm{e}^{-n},
$$

using $1+x \leq \mathrm{e}^{x}$ for $x \in \mathbb{R}$. So the probability that $A$ is not a covering code is at most $2^{n} \mathrm{e}^{-n}$, which tends to 0 as $n \rightarrow \infty$.

This procedure can be de-randomized by taking in each step a new code word $a \in\{0,1\}^{n}$ that is best possible in the sense that it covers as many as possible of the yet uncovered elements in $\{0,1\}^{n}$. Note, however, that this greedy construction takes $\mathcal{O}^{*}\left(2^{n}\right)$ per step and thus almost $\mathcal{O}\left(2^{2 n}\right)=\mathcal{O}^{*}\left(4^{n}\right)$ in total (which is far too slow). Dantsin et. al. [1] therefore propose the following. Let 
$K \in \mathbb{N}$ be a constant and assume w.l.o.g. that $n=K n_{0}$ and $r=K r$. Then construct a covering code $A_{0} \subseteq\{0,1\}^{n_{0}}$ in time $\mathcal{O}\left(4^{n_{0}}\right)=\mathcal{O}^{*}\left(\sqrt[K]{4^{n}}\right)$ and take

$$
A=\underbrace{A_{0} \times \ldots \times A_{0}}_{K \text { times }}
$$

as a covering code for $\{0,1\}^{n}$. Proceeding this way, the time needed for constructing the covering code becomes negligible.

Local search. Assume we want to search for a truth assignment for $\varphi$ in $B_{r}(a) \subseteq\{0,1\}^{n}$. We may assume w.l.o.g. that $a=0$, i.e., we search in $B_{r}=$ $B_{r}(0)$. (Interchange $x_{i}$ with $\bar{x}_{i}$ if necessary.) If $a=0$ is not a truth assignment for $\varphi$, there must exist a false clause, i.e. a clause $C \in \mathcal{C}$ that is false under $a=0$, say $C=\left(x_{i} \vee x_{i^{\prime}} \vee x_{i^{\prime \prime}}\right)$. It then suffices to search for a truth assignment in $B_{r-1} \subseteq\{0,1\}^{n-1}$ w.r.t. each of the formulae

$$
\varphi_{1}=\varphi\left[x_{i}=1\right], \varphi_{2}=\varphi\left[x_{i^{\prime}}=1\right] \text { and } \varphi_{3}=\varphi\left[x_{i^{\prime \prime}}=1\right],
$$

obtained by fixing a variable as indicated in brackets. If necessary, we may even fix in addition some variables to zero, e.g., define $\varphi_{1}:=\varphi\left[x_{i}=1\right], \varphi_{2}:=$ $\varphi\left[x_{i^{\prime}}=1, x_{i}=0\right]$ and $\varphi_{3}:=\varphi\left[x_{i^{\prime \prime}}=1, x_{i}=0, x_{i^{\prime}}=0\right]$.

Continuing this way, our search can be described by a search tree $T_{r}$, constructed by branching on false clauses (one false clause per node), as indicated in figure 1.

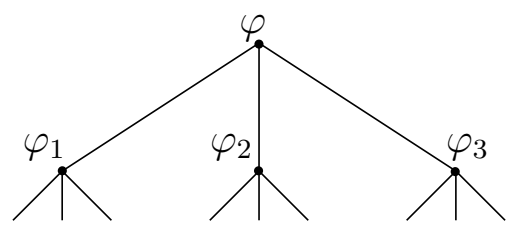

Fig. 1. The search tree $T_{r}$

Needless to say that we never branch to formulas $\varphi^{\prime}=\varphi\left[x_{i}=1, \ldots\right]$ that are obviously non-satisfiable because they contain an empty (non-satisfiable) clause. (For example, if $\left(\bar{x}_{i}\right) \in \mathcal{C}$, we would only branch to $\varphi_{2}$ and $\varphi_{3}$ in figure 1.) We denote the number of leaves of $T_{r}$ by $\left|T_{r}\right|$ and refer to it as the size of $T_{r}$. Clearly,

$$
\left|T_{r}\right| \leq 3^{r}
$$

holds, an immediate consequence of the recursion $\left|T_{r}\right| \leq 3\left|T_{r-1}\right|$ (see figure 1). In case $\varphi$ contains a false 2-clause $C \in \mathcal{C}$, then branching on $C$ would yield $\left|T_{r}\right| \leq 2\left|T_{r-1}\right|$.

As pointed out in Dantsin et. al. [1], this simple argument already gives an $\mathcal{O}^{*}\left(\sqrt[2]{3}^{n}\right) \approx \mathcal{O}^{*}\left(1.7321^{n}\right)$ algorithm: Take $r=\frac{n}{2}$ and search $B_{r}(0)$ and $B_{r}(1)$ 
separately in time $\mathcal{O}^{*}\left(3^{r}\right)=\mathcal{O}^{*}\left(\sqrt[2]{3}^{n}\right)$ each.

Smaller search trees. The trivial bound (1) on the size of the search tree can be improved by a clever branching technique, as shown in Dantsin et. al. [1]: Assume that $\varphi$ contains three pairwise disjoint false clauses $C=$ $\left(x_{i} \vee x_{i^{\prime}} \vee x_{i^{\prime \prime}}\right), C_{1}=\left(x_{j} \vee x_{j^{\prime}} \vee x_{j^{\prime \prime}}\right)$ and $C_{1}^{\prime}=\left(x_{k} \vee x_{k^{\prime}} \vee x_{k^{\prime \prime}}\right)$ and a (true) clause $\left(\bar{x}_{i} \vee \bar{x}_{j} \vee \bar{x}_{k}\right)$. We may then branch along $\left(\bar{x}_{i} \vee \bar{x}_{j} \vee \bar{x}_{k}\right)$, i.e. first branch on $C$ at the root node $\varphi$, then branch on $C_{1}$ at $\varphi_{1}=\varphi\left[x_{i}=1\right]$ and finally branch on $C_{1}^{\prime}$ at $\varphi_{1}^{\prime}=\varphi_{1}\left[x_{j}=1\right]=\varphi\left[x_{i}=1, x_{j}=1\right]$. The resulting search tree is indicated in figure 2 .

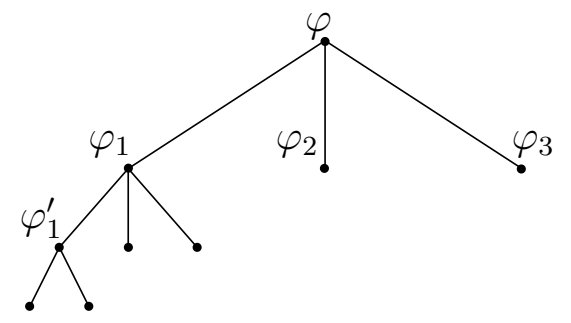

Fig. 2. Branching along $\left(\bar{x}_{i} \vee \bar{x}_{j} \vee \bar{x}_{k}\right)$

Note that the node corresponding to $\varphi_{1}^{\prime}$ has only two descendants because $\varphi\left[x_{i}=1, x_{j}=1, x_{k}=1\right]$ is ruled out by the clause $\left(\bar{x}_{i} \vee \bar{x}_{j} \vee \bar{x}_{k}\right)$.

If a similar branching was possible also at $\varphi_{2}$ and $\varphi_{3}$, we would get a search tree satisfying a recursion

$$
\left|T_{r}\right| \leq 6\left|T_{r-2}\right|+6\left|T_{r-3}\right|
$$

Indeed, this is what Dantsin et. al. [1] show. Assuming inductively that $\left|T_{k}\right| \leq$ $c \alpha^{k}$ holds for some constant $c>0,(2)$ implies that

$$
\left|T_{r}\right| \leq \mathcal{O}\left(\alpha^{r}\right)
$$

where $\alpha=\sqrt[3]{4}+\sqrt[3]{2} \approx 2.848$ is the largest root of $\alpha^{3}-6 \alpha-6=0$.

The main result of our paper slightly improves this bound as follows.

Theorem 1 By branching on false clauses we can ensure that

$$
\left|T_{r}\right| \leq c \beta^{r}
$$

where $\beta=\frac{1+\sqrt{21}}{2} \approx 2.792$ is the largest root of $\beta^{3}-6 \beta-5=0$. 
Running time. Let $\varrho<\frac{1}{2}$ and $r=\varrho n$. By Stirling's formula, the size of a covering code we construct is (up to a polynomial factor) bounded by

$$
|A|=\mathcal{O}^{*}\left(\left[2 \varrho^{\varrho}(1-\varrho)^{1-\varrho}\right]^{n}\right) \text {. }
$$

According to (3), the number of nodes in $T_{r}$ is bounded by $n\left|T_{r}\right|=\mathcal{O}^{*}\left(\left|T_{r}\right|\right)$ and hence the total running time is thus bounded by

$$
\mathcal{O}^{*}\left(|A|\left|T_{r}\right|\right)=\mathcal{O}^{*}\left(\left[2(\alpha \varrho)^{\varrho}(1-\varrho)^{1-\varrho}\right]^{n}\right) .
$$

This expression is minimal for $\varrho \approx 0.26$, yielding the bound of $\mathcal{O}^{*}\left(1.481^{n}\right)$ in Dantsin et. al. [1].

Similarly, replacing $\alpha$ by $\beta$ from Theorem 1 , we obtain for $\varrho \approx 0.264$ an exact algorithm that runs in $\mathcal{O}^{*}\left(1.473^{n}\right)$.

\section{Simple partial assignments}

We will prove Theorem 1 by induction on $r \geq 0$. The basic idea is as follows. We first try to find a "simple truth assignment" by fixing as few as possible of the variables to $x_{i}=1$ (exactly one per false clause). In case we do not succeed, we will exhibit a "good" clause to branch on.

We start by analyzing the structure of $\mathcal{C}$ and introduce some notation. Let $\mathcal{F} \subseteq \mathcal{C}$ denote the set of false clauses (at $x=0$ ). We may assume w.l.o.g. that each $F \in \mathcal{F}$ is a 3-clause $F=\left(x_{i} \vee x_{i^{\prime}} \vee x_{i^{\prime \prime}}\right)$, because otherwise, as we observed already in section 2 , branching on a false clause of length at most 2 yields the recursion $\left|T_{r}\right| \leq 2\left|T_{r-1}\right|$ and Theorem 1 follows by induction.

Secondly, we may assume that the clauses $F \subseteq \mathcal{F}$ are pairwise disjoint. Indeed, if $F=\left(x_{i} \vee x_{i^{\prime}} \vee x_{i^{\prime \prime}}\right)$ and $F^{\prime}=\left(x_{j} \vee x_{j^{\prime}} \vee x_{j^{\prime \prime}}\right)$ intersect, say $x_{i}=x_{j}$, then branching on $F$ at $\varphi$ and on $F^{\prime}$ at $\varphi_{2}=\varphi\left[x_{i^{\prime}}=1, x_{i}=0\right]$ and $\varphi_{3}=$ $\varphi\left[x_{i^{\prime \prime}}=1, x_{i}=0, x_{i^{\prime}}=0\right]$ yields a search tree as indicated in figure 3 .

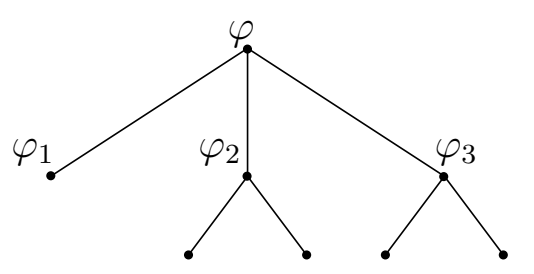

Fig. 3. Branching on intersecting clauses

The corresponding recursion is $\left|T_{r}\right| \leq\left|T_{r-1}\right|+4\left|T_{r-2}\right|$ and, again, Theorem 1 follows inductively. 
Thus in what follows, we may (and will) assume that $\varphi$ is regular in the sense that $\mathcal{F}$ consists of pairwise disjoint 3-clauses. We often identify such a clause $F=\left(x_{i} \vee x_{i^{\prime}} \vee x_{i^{\prime \prime}}\right) \in \mathcal{F}$ with its corresponding set of variables $F=$ $\left\{x_{i}, x_{i^{\prime}}, x_{i^{\prime \prime}}\right\}$ or with the corresponding set of elements (indices) $F=\left\{i, i^{\prime}, i^{\prime \prime}\right\}$. The elements $i, i^{\prime}, i^{\prime \prime}$ covered by a false clause $F \in \mathcal{F}$ are neighbors of each other. The elements $i \in\{1, \ldots, n\}$ covered by false clauses are called internal elements. We denote by $I=I_{\varphi} \subseteq\{1, \ldots, n\}$ the set of internal elements. The elements in $\{1, \ldots, n\} \backslash I$ are called external.

Recall that, as mentioned above, we first try to construct a truth assignment for $\varphi$ by fixing some variable to $x_{i}=1$ (one per false clause in $\mathcal{F}$ ). In general, fixing some variables, say $x_{i_{1}}=1, \ldots, x_{i_{t}}=1$, results in a new formula $\varphi^{\prime}=$ $\varphi\left[x_{i_{1}}=1, \ldots, x_{i_{t}}=1\right]$ whose clauses are obtained from the clauses in $\mathcal{C}$ by fixing $x_{i_{1}}=1, \ldots, x_{i_{t}}=1$ in each clause. This way each clause $C \in \mathcal{C}$ reduces to a corresponding clause $C^{\prime}=C\left[x_{i_{1}}=1, \ldots, x_{i_{t}}=1\right] \in \mathcal{C}^{\prime}=\mathcal{C}_{\varphi^{\prime}}$. We say that $C$ reduces to $C^{\prime}=1$ (a fixed true clause) if $C$ contains some $x_{i}, i \in\left\{i_{1}, \ldots, i_{t}\right\}$. Similarly, $C$ reduces to $C^{\prime}=0$, the empty (fixed false) clause if $C$ contains only negated literals $\bar{x}_{i}, i \in\left\{i_{1}, \ldots, i_{t}\right\}$. Note that $C \in \mathcal{C}$ reduces to $C^{\prime} \in \mathcal{F}_{\varphi^{\prime}}$ if and only if all negated variables $\bar{x}_{i}$ in $C$ are indexed by $i \in\left\{i_{1}, \ldots, i_{t}\right\}$.

Definition 2 (Simple partial assignment) $A$ simple partial assignment (SPA) of $\varphi$ is a formula

$$
\varphi^{\prime}=\varphi\left[x_{i_{1}}=1, \ldots, x_{i_{t}}=1\right]
$$

that fixes at most one variable per false clause to $x_{i}=1$, without creating any new false clauses, i.e., such that the following hold:

$$
\begin{aligned}
& (S 1)\left\{i_{1}, \ldots, i_{t}\right\} \subseteq I \\
& (S 2)\left|F \cap\left\{i_{1}, \ldots, i_{t}\right\}\right| \leq 1 \text { for each } F \in \mathcal{F}_{\varphi} \\
& (S 3) \mathcal{F}_{\varphi^{\prime}} \subseteq \mathcal{F}_{\varphi} .
\end{aligned}
$$

There are certain clauses in $\mathcal{C} \backslash \mathcal{F}$ that are "irrelevant" in the sense that they never reduce to a false clause by fixing $x_{i_{1}}=1, \ldots, x_{i_{t}}=1$ as long as (S1) and (S2) hold: A clause $C \in \mathcal{C} \backslash \mathcal{F}$ is called externally true if $C=\left(\bar{x}_{l} \vee \ldots\right)$ with $l \in\{1, \ldots, n\} \backslash I$ being external. A clause $C \in \mathcal{C} \backslash \mathcal{F}$ is internally true if $C=\left(\bar{x}_{i} \vee \bar{x}_{j} \vee \ldots\right)$ with $i, j \in I$ being neighbors. Clearly, an externally and/or internally true $C \in \mathcal{C}$ reduces to a true clause $C^{\prime} \in \mathcal{C}_{\varphi^{\prime}}$ whenever $\varphi^{\prime}=\varphi\left[x_{i_{1}}=1, \ldots, x_{i_{t}}=1\right]$ satisfies $(\mathrm{S} 1)$ and $(\mathrm{S} 2)$. We let $\mathcal{E} \subseteq \mathcal{C} \backslash \mathcal{F}$ denote the set of externally and/or internally true clauses.

The remaining set $\mathcal{R}=\mathcal{C} \backslash(\mathcal{F} \cup \mathcal{E})$ is called the set of relevant clauses. We will use these clauses to guide our search process, i.e., we will construct $T_{r}$ by "branching along relevant clauses" as indicated already in section 2 . We 
first treat the so-called "pure case", where each relevant clause contains only negated variables. This is the case where the bound (2) is tight in the approach of Dantsin et. al. [1].

\section{The pure case}

A regular $\varphi$ is called pure if every $R \in \mathcal{R}=\mathcal{R}_{\varphi}$ contains only negated variables. Throughout this section, we assume that $\varphi$ is (regular and) pure and hence so is any $S P A \varphi^{\prime}$ of $\varphi$.

We say that $R \in \mathcal{R}$ intersects $F=\left(x_{i} \vee x_{i^{\prime}} \vee x_{i^{\prime \prime}}\right) \in \mathcal{F}$ if $R$ contains one of $\bar{x}_{i}, \bar{x}_{i^{\prime}}, \bar{x}_{i^{\prime \prime}}$. Recall that $R$ cannot contain two of these since it would then be internally true. To motivate the following, consider an $S P A \varphi^{\prime}=\varphi\left[x_{i}=1\right]$ of $\varphi$. Any $R \in \mathcal{R}$ reduces to a true clause in $\varphi^{\prime}$ due to (S3). If $R$ intersects the unique false clause $F=\left(x_{i} \vee x_{i^{\prime}} \vee x_{i^{\prime \prime}}\right)$ covering $i$, then either $R$ becomes an externally true clause in $\varphi^{\prime}$ (namely when $R$ contains either $\bar{x}_{i^{\prime}}$ or $\bar{x}_{i^{\prime \prime}}$ ) or $R$ reduces to an "even more" relevant clause $R^{\prime} \in \mathcal{R}_{\varphi^{\prime}}$. For example, $R=$ $\left(\bar{x}_{i} \vee \bar{x}_{j} \vee \bar{x}_{k}\right)$ reduces to $R^{\prime}=\left(\bar{x}_{j} \vee \bar{x}_{k}\right) \in \mathcal{R}_{\varphi^{\prime}}$.

Let $\varphi^{\prime}=\varphi\left[x_{i_{1}}=1, \ldots, x_{i_{t}}=1\right]$ be an $S P A$ of $\varphi$ and let $F_{i_{1}}, \ldots, F_{i_{t}} \in \mathcal{F}$ be the unique clauses covering $i_{1}, \ldots, i_{t}$, resp. We say that $\varphi^{\prime}$ is proper if every $R \in \mathcal{R}$ that intersects some $F \in\left\{F_{i_{1}}, \ldots, F_{i_{t}}\right\}$ reduces to an externally true clause $R^{\prime} \in \mathcal{R}_{\varphi^{\prime}}$ (so $R$ must contain some $\bar{x}_{i}$ with $i \in I$ being a neighbor of an element in $\left.\left\{i_{1}, \ldots, i_{t}\right\}\right)$.

Lemma 3 For any two proper SPA's $\varphi^{\prime}$ and $\varphi^{\prime \prime}$ of $\varphi$ there exist a proper SPA $\bar{\varphi}$ with $\mathcal{F}_{\bar{\varphi}}=\mathcal{F}_{\varphi^{\prime}} \cap \mathcal{F}_{\varphi^{\prime \prime}}$.

PROOF. Let $\mathcal{F}_{\varphi}=\left\{F_{1}, \ldots, F_{f}\right\}$ with $F_{i}=\left(x_{i} \vee x_{i^{\prime}} \vee x_{i^{\prime \prime}}\right), i=1, \ldots, f$, and assume that, say,

$$
\begin{aligned}
\varphi^{\prime} & =\varphi\left[x_{1}=1, \ldots, x_{s}=1\right] \\
\varphi^{\prime \prime} & =\varphi\left[x_{s+1}=1, \ldots, x_{t}=1, x_{j_{1}}=1, \ldots, x_{j_{l}}=1\right]
\end{aligned}
$$

with $j_{1}, \ldots, j_{l}$ being covered by $F_{1}, \ldots, F_{s}$. We define $\bar{\varphi}$ as

$$
\bar{\varphi}=\varphi\left[x_{1}=1, \ldots, x_{t}=1\right] .
$$

Clearly, $\bar{\varphi}$ satisfies (S1) and (S2). We verify (S3) by showing that any $R \in \mathcal{R}_{\varphi}$ reduces to a true clause $\bar{R} \in \mathcal{R}_{\bar{\varphi}}$. Indeed, we will show that any $R \in \mathcal{R}_{\varphi}$ intersecting $F_{1} \cup \ldots \cup F_{t}$ reduces (even) to an externally true clause in $\bar{\varphi}$, thus showing at the same time that $\bar{\varphi}$ is proper. 
Let $R \in \mathcal{R}_{\varphi}$ intersect $F_{i} \in\left\{F_{1}, \ldots, F_{t}\right\}$. If $i \leq s$, then $R$ reduces to an externally true clause in $\varphi^{\prime}$ (since $\varphi^{\prime}$ is proper) and hence to an externally true clause in $\bar{\varphi}$. On the other hand, if $R$ does not intersect $F_{1} \cup \ldots \cup F_{s}$ (but $F_{s+1} \cup \ldots \cup F_{t}$ ), then $R$ reduces to the same clause in $\bar{\varphi}$ as in $\varphi^{\prime \prime}$. So again, the claim follows, as $\varphi^{\prime \prime}$ is proper.

Lemma 3 is useful in constructing proper $S P A$ 's $\bar{\varphi}$ with smaller and smaller sets $\mathcal{F}_{\bar{\varphi}}$. Ideally, we would like to arrive at $\mathcal{F}_{\bar{\varphi}}=\emptyset$, in which case $\bar{\varphi}$ defines a truth assignment for $\varphi$. To describe our search process for proper SPA's of $\varphi$, we introduce the notion of "b-blocking".

Definition 4 (b-blocking) Consider a clause $R \in \mathcal{R}_{\varphi}$.

(1) If $R=\left(\bar{x}_{i} \vee \ldots\right)$ then $R$ 0-blocks $i \in I$.

(2) If $R=\left(\bar{x}_{i} \vee \ldots\right)$ has length at most two, then $R b$-blocks $i$ for all $b \geq 0$.

(3) If $R=\left(\bar{x}_{i} \vee \ldots\right)$ has length three, i.e. $R=\left(\bar{x}_{i} \vee \bar{x}_{j} \vee \bar{x}_{k}\right)$ for some $j, k \in I$ with neighbors $j^{\prime}, j^{\prime \prime}$ and $k^{\prime}, k^{\prime \prime}$, resp., then $R$-blocks $i$, if each of $j^{\prime}, j^{\prime \prime}, k^{\prime}$ and $k^{\prime \prime}$ is $(b-1)$-blocked by some clause in $\mathcal{R}_{\varphi\left[x_{i}=1\right]}$.

We call $i \in I$ b-blocked by $\mathcal{R}_{\varphi}$ if there exists some $R \in \mathcal{R}_{\varphi}$ (of arbitrary length) that b-blocks $i$.

Example. Assume $\mathcal{F}=\mathcal{F}_{\varphi}$ consists of three clauses $\left(x_{i} \vee x_{i^{\prime}} \vee x_{i^{\prime \prime}}\right)$, $\left(x_{j} \vee x_{j^{\prime}} \vee x_{j^{\prime \prime}}\right)$ and $\left(x_{k} \vee x_{k^{\prime}} \vee x_{k^{\prime \prime}}\right)$. Furthermore, assume that $\mathcal{R}=\mathcal{R}_{\varphi}$ consists of three clauses $R=\left(\bar{x}_{i} \vee \bar{x}_{j} \vee \bar{x}_{k}\right), R^{\prime}=\left(\bar{x}_{i^{\prime}} \vee \bar{x}_{j^{\prime}} \vee \bar{x}_{k^{\prime}}\right)$ and $R^{\prime \prime}=$ $\left(\bar{x}_{i^{\prime \prime}} \vee \bar{x}_{j^{\prime \prime}} \vee \bar{x}_{k^{\prime \prime}}\right)$. Then each element in $I=I_{\varphi}$ is 0-blocked, but none is 1-blocked. Indeed, consider, e.g. $\varphi^{\prime}=\varphi\left[x_{i}=1\right]$. Then $R^{\prime}$ and $R^{\prime \prime}$ reduce to externally true clauses in $\varphi^{\prime}$. So $\mathcal{R}_{\varphi^{\prime}}=\left\{\left(\bar{x}_{j} \vee \bar{x}_{k}\right)\right\}$ and, for example, $j^{\prime}$ is not 0 -blocked by $\mathcal{R}_{\varphi^{\prime}}$. For this reason (see the general construction described below), it is easy to find a truth assignment for $\varphi$ (e.g. by setting $\left.x_{i}=1, x_{j^{\prime}}=1, x_{k^{\prime}}=1\right)$.

For $b \geq 0$, we let $U_{b} \subseteq I$ denote the set of elements $i \in I$ that are not $b$-blocked by $\mathcal{R}_{\varphi}$. We call these elements $b$-unblocked (by $\mathcal{R}_{\varphi}$ ). Let $\mathcal{U}_{b} \subseteq \mathcal{F}$ denote the set of false clauses $F \in \mathcal{F}$ that cover some $b$-unblocked $i \in I$. We also call these false clauses $b$-unblocked. By definition, we have $U_{0} \subseteq U_{1} \subseteq \ldots$ and also $\mathcal{U}_{0} \subseteq \mathcal{U}_{1} \subseteq \ldots$

Note that we can compute the set $U_{b} \subseteq I$ for $b \geq 0$ along with a $b$-blocking clause $R \in \mathcal{R}_{\varphi}$ for every $i \in I \backslash U_{b}$ in time $\mathcal{O}\left(n^{b+3}\right)$. Indeed, for $b=0$, it suffices to scan the $\mathcal{O}\left(n^{3}\right)$ clauses in $\mathcal{R}=\mathcal{R}_{\varphi}$. 
We proceed by induction on $b \geq 0$. Thus assume $b \geq 1$ and let $i \in I$ and $\varphi^{\prime}=\varphi\left[x_{i}=1\right]$. By induction, the set $U_{b-1}^{\prime} \subseteq I_{\varphi^{\prime}}$ of elements that are $(b-$ 1)-unblocked by $\mathcal{R}_{\varphi^{\prime}}$ can be computed in time $\mathcal{O}\left(n^{b+2}\right)$. We then check for each of the $\mathcal{O}\left(n^{2}\right)$ 3-clauses $R=\left(\bar{x}_{i} \vee \bar{x}_{j} \vee \bar{x}_{k}\right)$ whether some element from $\left\{j^{\prime}, j^{\prime \prime}, k^{\prime}, k^{\prime \prime}\right\}$ is in $U_{b-1}^{\prime}$ or not. This takes (at most) $\mathcal{O}\left(n^{2}\right) \mathcal{O}(n)=\mathcal{O}\left(n^{3}\right)$ in total. Hence the total time needed to check $i \in I$ is $\mathcal{O}\left(n^{b+2}\right)+\mathcal{O}\left(n^{3}\right)=$ $\mathcal{O}\left(n^{b+2}\right)$ and the claim follows.

The next result is crucial:

Theorem 5 For each $b \geq 0$ there exists a proper SPA $\varphi^{\prime}$ of $\varphi$ with $\mathcal{F}_{\varphi^{\prime}} \subseteq$ $\mathcal{F}_{\varphi} \backslash \mathcal{U}_{b}$

PROOF. By induction on $b \geq 0$. Assume first that $b=0$. Let $F \in \mathcal{U}_{0}$, say $F=\left(x_{i} \vee x_{i^{\prime}} \vee x_{i^{\prime \prime}}\right)$ with $i \in U_{0}$. Then $\varphi^{\prime}=\varphi\left[x_{i}=1\right]$ is, by definition of $U_{0}$, a proper $S P A$ and $\mathcal{F}_{\varphi^{\prime}}=\mathcal{F} \backslash\{F\}$. The claim now follows from Lemma 3 and induction.

Next assume $b \geq 1$. Let $F=\left(x_{i} \vee x_{i^{\prime}} \vee x_{i^{\prime \prime}}\right) \in \mathcal{U}_{b}$ with $i \in U_{b}$. As before, due to Lemma 3, it suffices to show that there is a proper $S P A \varphi^{\prime}$ of $\varphi$ with $\mathcal{F}_{\varphi^{\prime}} \subseteq \mathcal{F} \backslash\{F\}$. Let $\varphi_{1}:=\varphi\left[x_{i}=1\right]$. Clearly, $\varphi_{1}$ is an $S P A$ of $\varphi$. (Otherwise there were a clause $\left(\bar{x}_{i}\right) \in \mathcal{R}$. But such a clause would $b$-block $i$ contradicting $i \in U_{b}$.) Let $U_{b-1}^{1} \subseteq I_{\varphi_{1}}$ and $\mathcal{U}_{b-1}^{1} \subseteq \mathcal{F}_{\varphi_{1}}$ denote the set of elements in $I_{\varphi_{1}}$ resp. clauses in $\mathcal{F}_{\varphi_{1}}$ that are $(b-1)$-unblocked by $\mathcal{R}_{\varphi_{1}}$. By induction on $b$, there is a proper $S P A \varphi_{1}^{\prime}$ of $\varphi_{1}$ with $\mathcal{F}_{\varphi_{1}^{\prime}} \subseteq \mathcal{F}_{\varphi_{1}} \backslash \mathcal{U}_{b-1}^{1}$. We claim that actually $\varphi_{1}^{\prime}$ is a proper $S P A$ of $\varphi$. Clearly, $\varphi_{1}^{\prime}$ is an $S P A$ of $\varphi$ (as any $S P A$ of an $S P A$ is an $S P A)$.

To show that $\varphi_{1}^{\prime}$ is proper, assume that

$$
\varphi_{1}^{\prime}=\varphi_{1}\left[x_{i_{1}}=1, \ldots, x_{i_{t}}=1\right]=\varphi\left[x_{i}=1, x_{i_{1}}=1, \ldots, x_{i_{t}}=1\right]
$$

and let $F_{i}, F_{i_{1}}, \ldots, F_{i_{t}} \in \mathcal{F}$ denote the unique clauses in $\mathcal{F}$ covering $i, i_{1}, \ldots, i_{t}$, resp. Let $R \in \mathcal{R}_{\varphi}$ intersect $F_{i} \cup F_{i_{1}} \cup \ldots \cup F_{i_{t}}$. We are to show that $R$ reduces to an externally true clause $R_{1}^{\prime}$ in $\varphi_{1}^{\prime}$.

Assume first that $R$ intersects $F_{i}=\left(x_{i} \vee x_{i^{\prime}} \vee x_{i^{\prime \prime}}\right)$. If $R$ contains either $\bar{x}_{i^{\prime}}$ or $\bar{x}_{i^{\prime \prime}}$, the claim is obviously true. Thus assume $R=\left(\bar{x}_{i} \vee \ldots\right) \in \mathcal{R}$. Since $i \in U_{b}$, $R$ must be a 3 -clause $R=\left(\bar{x}_{i} \vee \bar{x}_{j} \vee \bar{x}_{k}\right)$. So $R$ reduces to $R_{1}=\left(\bar{x}_{j} \vee \bar{x}_{k}\right)$ in $\varphi_{1}$. As $i \in U_{b}$, at least one neighbor of either $j$ or $k$ is in $\mathcal{U}_{b-1}^{1}$, i.e., either $F_{j}=$ $\left(x_{j} \vee x_{j^{\prime}} \vee x_{j^{\prime \prime}}\right)$ or $F_{k}=\left(x_{k} \vee x_{k^{\prime}} \vee x_{k^{\prime \prime}}\right)$ is in $\mathcal{U}_{b-1}^{1} \subseteq \mathcal{F}_{\varphi_{1}}$. So $\mathcal{F}_{\varphi_{1}^{\prime}} \subseteq \mathcal{F}_{\varphi_{1}} \backslash \mathcal{U}_{b-1}^{1}$ implies that $\varphi_{1}^{\prime}$ fixes at least one variable from either $F_{j}$ or $F_{k}$ to 1, i.e., either $F_{j}$ or $F_{k}$ occurs in $\left\{F_{i_{1}}, \ldots, F_{i_{t}}\right\}$. Thus $R_{1}=\left(\bar{x}_{j} \vee \bar{x}_{k}\right)$ reduces to an externally true clause $R_{1}^{\prime}$ in $\varphi_{1}^{\prime}$ (as $\varphi_{1}^{\prime}$ is a proper $S P A$ of $\varphi$ ) and hence so does $R$. 
Next assume that $R$ does not intersect $F_{i}$. Then $R \in \mathcal{R}_{\varphi}$ and the claim follows immediately from the fact that $\varphi_{1}^{\prime}$ is a proper $S P A$ of $\varphi_{1}$.

Corollary 6 If $\mathcal{U}_{b}=\mathcal{F}$ for some $b \geq 0$, then $\varphi$ has a truth assignment that can be computed in time $\mathcal{O}\left(n^{b+3}\right)$.

We are now ready to prove Theorem 1 in the pure case. Let $b \geq 0$ (to be specified later on) and assume there exists some $F=\left(x_{i} \vee x_{i^{\prime}} \vee x_{i^{\prime \prime}}\right) \in \mathcal{F} \backslash \mathcal{U}_{b}$. (Otherwise a truth assignment exists and there is no need to construct a search tree.) We then branch on $F$ at the root node $\varphi$ of $T_{r}$, branching to $\varphi_{1}=\varphi\left[x_{i}=1\right], \varphi_{2}=\varphi\left[x_{i^{\prime}}=1\right]$ and $\varphi_{3}=\varphi\left[x_{i^{\prime \prime}}=1\right]$.

Since $F \notin \mathcal{U}_{b}$, the elements $i, i^{\prime}$ and $i^{\prime \prime}$ are $b$-blocked by $\mathcal{R}_{\varphi}$. Let $R \in \mathcal{R} b$-block $i$. If $R$ is a 1-clause, i.e. $R=\left(\bar{x}_{i}\right)$, then the subtree rooted at $\varphi_{1}$ is empty. If $R$ is a 2-clause, i.e. $R=\left(\bar{x}_{i} \vee \bar{x}_{j}\right)$, then branching on $F_{1}=\left(x_{j} \vee x_{j^{\prime}} \vee x_{j^{\prime \prime}}\right)$ at $\varphi_{1}$ yields a search tree as indicated in figure 4 . Thus we obtain a recursion $\left|T_{r}\right| \leq 2\left|T_{r-1}\right|+2\left|T_{r-2}\right|$ and Theorem 1 follows inductively.

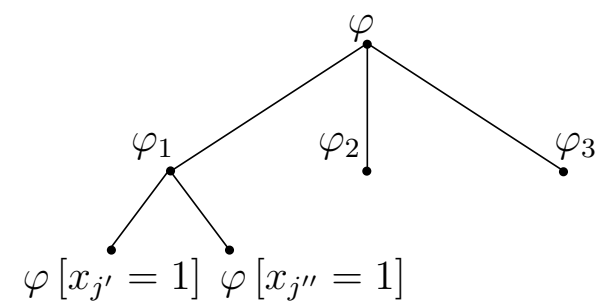

Fig. 4. When $i$ is blocked by a 2 -clause.

Hence assume that $R=\left(\bar{x}_{i} \vee \bar{x}_{j} \vee \bar{x}_{k}\right)$ b-blocks $i$. In this case we obtain a search tree as in figure 2 by branching on $F_{1}$ at $\varphi_{1}$ and on $F_{1}^{\prime}=\left(x_{k} \vee x_{k^{\prime}} \vee x_{k^{\prime \prime}}\right)$ at $\varphi_{1}^{\prime}=\varphi_{1}\left[x_{j}=1\right]$.

Let us denote the size of the subtree rooted at $\varphi_{1}$ by $\left|T_{r-1}^{(b)}\right|$ to indicate that $\varphi_{1}=\varphi\left[x_{i}=1\right]$ is obtained by fixing $x_{i}$ with $i$ being $b$-blocked by $\mathcal{R}_{\varphi}$. We thus get the recursion

$$
\left|T_{r-1}^{(b)}\right| \leq 2\left|T_{r-2}^{(b-1)}\right|+2\left|T_{r-3}\right|,
$$

as both $j^{\prime}$ and $j^{\prime \prime}$ are $(b-1)$-blocked by $\mathcal{R}_{\varphi_{1}}$. Furthermore, of course $\left|T_{r}\right| \leq$ $3\left|T_{r-1}^{(b)}\right|$ holds, since also $i^{\prime}$ and $i^{\prime \prime}$ are $b$-blocked by $\mathcal{R}_{\varphi}$.

Iterating (4), we obtain for $r \geq b+2$ 


$$
\begin{aligned}
\left|T_{r-1}^{(b)}\right| & \leq 2\left[2\left|T_{r-3}^{(b-2)}\right|+2\left|T_{r-2}\right|\right]+2\left|T_{r-3}\right| \\
& \vdots \\
& \leq 2^{b}\left|T_{r-b-2}\right|+\ldots+2\left|T_{r-3}\right|+2^{b}\left|T_{r-b-1}^{(0)}\right| \\
& \leq 2^{b}\left|T_{r-b-2}\right|+\ldots+2\left|T_{r-3}\right|+2^{b}\left|T_{r-b-1}\right|
\end{aligned}
$$

where the last inequality follows from $\left|T_{k}^{(0)}\right| \leq\left|T_{k}\right|$.

Assuming inductively that $\left|T_{k}\right| \leq c \beta^{k}$ for $k<r$, we get

$$
\begin{aligned}
\left|T_{r}\right| & \leq 3\left|T_{r}^{(b-1)}\right| \\
& \leq 3 c \beta^{r}\left[\frac{2^{b}}{\beta^{b+1}}+\sum_{k=1}^{b} \frac{2^{k}}{\beta^{k+2}}\right] \\
& =3 c \beta^{r}\left[\frac{2^{b}}{\beta^{b+1}}+\frac{2-2^{b+1} \beta^{-b}}{\beta^{3}-2 \beta^{2}}\right] .
\end{aligned}
$$

For $\beta$ as in Theorem 1 and $b \geq 4$ we have for the term in the brackets

$$
\frac{2^{b}}{\beta^{b+1}}+\frac{2-2^{b+1} \beta^{-b}}{\beta^{3}-2 \beta^{2}}<\frac{1}{3} \text {. }
$$

So $\left|T_{r}\right| \leq c \beta^{r}$ follows inductively.

\section{The general case}

In the general case, when $\varphi$ is regular, but not necessarily pure, we proceed as follows. As in section 4 we say that $i \in I$ is blocked by $R \in \mathcal{R}$ if $R=\left(\bar{x}_{i} \vee \ldots\right)$. Let $U \subseteq I$ denote the elements that are unblocked, i.e. not blocked by any $R \in \mathcal{R}$ and let $\mathcal{U} \subseteq \mathcal{F}$ denote the set of clauses $F \in \mathcal{F}$ that contain some $i \in U$.

If $\mathcal{F}=\mathcal{U}$, a truth assignment is easily obtained by fixing exactly one unblocked $i$ per clause $F \in \mathcal{F}$ to $x_{i}=1$. Hence assume $\mathcal{F}^{*}=\mathcal{F} \backslash \mathcal{U} \neq \emptyset$ in what follows and let $I^{*} \subseteq I$ denote the elements covered by clauses in $\mathcal{F}^{*}$. We distinguish two cases:

Case 1. There exists an element $i \in I^{*}$ that is blocked by some $R \in \mathcal{R}$ which is not of the form $R=\left(\bar{x}_{i} \vee \bar{x}_{j} \vee \bar{x}_{k}\right)$ with $j, k \in I$.

In this case we branch on the unique clause $F \in \mathcal{F}^{*}$ covering $i$. Branching along blocking clauses as in section 4 then proves Theorem 1 inductively. 
Indeed, assume that $i$ is blocked by a clause of type $R=\left(\bar{x}_{i} \vee \bar{x}_{j} \vee x_{k}\right)$ with $j, k \in I$. Note that $j$ is then covered by a clause $F_{1} \neq F$ since otherwise $R$ were internally true. We then branch on $F_{1}=\left(x_{j} \vee x_{j^{\prime}} \vee x_{j^{\prime \prime}}\right)$ at $\varphi_{1}=\varphi\left[x_{i}=1\right]$ and on the false 1-clause $\left(x_{k}\right)$ at $\varphi_{1}^{\prime}=\varphi_{1}\left[x_{j}=1\right]$. The resulting search tree then differs from the one in figure 2 in that one of the two subtrees of $\varphi_{1}^{\prime}$ is eliminated, yielding a recursion

$$
\left|T_{r}\right| \leq 6\left|T_{r-2}\right|+5\left|T_{r-3}\right|
$$

assuming the "worst case scenario", where both $i^{\prime}$ and $i^{\prime \prime}$ are blocked by 3clauses with three negated variables each. In this case, Theorem 1 follows inductively (by choice of $\beta$ ). It is straightforward to verify that this is indeed the worst case scenario for case 1).

Case 2. All blocking clauses for elements in $I^{*}$ have three negated variables each.

In this case, let $\mathcal{R}^{*}$ denote the set of clauses $R=\left(\bar{x}_{i} \vee \bar{x}_{j} \vee \bar{x}_{k}\right) \in \mathcal{R}$ with $i, j, k \in I^{*}$. Let $\varphi^{*}$ denote the formula defined by the clauses $\mathcal{C}^{*}=\mathcal{F}^{*} \cup \mathcal{R}^{*}$. In particular, $\varphi^{*}$ is pure. Let $\mathcal{U}_{b}^{*} \subseteq \mathcal{F}$ denote the clauses in $\mathcal{F}^{*}$ that are $b$ unblocked by $\mathcal{R}_{\varphi^{*}}$.

Lemma 7 If $\mathcal{U}_{b}^{*}=\mathcal{F}^{*}$, then $\varphi$ has a truth assignment.

PROOF. By Theorem 5, $\varphi^{*}$ has a proper $S P A$

$$
\varphi^{\prime}=\varphi^{*}\left[x_{i_{1}}=1, \ldots, x_{i_{t}}=1\right]
$$

defining a truth assignment for $\varphi^{*}$ (see also Corollary 6).

To define a truth assignment for $\varphi$, pick elements $j_{1}, \ldots, j_{s} \in U$, one from each clause in $\mathcal{U}$, and let

$$
\bar{\varphi}=\varphi\left[x_{i_{1}}=1, \ldots, x_{i_{t}}=1, x_{j_{1}}=1, \ldots, x_{j_{s}}=1\right] .
$$

We claim that $\bar{\varphi}$ defines a truth assignment for $\varphi$, i.e. that $\mathcal{F}_{\bar{\varphi}}=\emptyset$. Assume to the contrary that $R \in \mathcal{R}$ reduces to a false clause in $\bar{\varphi}$. Clearly, $R \notin \mathcal{R}^{*}$ must hold, since any clause in $\mathcal{R}^{*}$ reduces to an (externally) true clause in $\varphi^{\prime}$ and hence to a true clause in $\bar{\varphi}$. However, if $R \in \mathcal{R} \backslash \mathcal{R}^{*}$, case 2) implies that $R=\left(\bar{x}_{i} \vee \ldots\right)$ with $i \in I \backslash I^{*}$. In particular, $i$ is blocked by $R$ and so $i \notin\left\{j_{1}, \ldots, j_{s}\right\}$. Thus, $R$ reduces to a true clause in $\bar{\varphi}$.

Due to Lemma 7 , we may assume w.l.o.g. that $\mathcal{U}_{b}^{*} \neq \mathcal{F}^{*}$. Thus we may choose $F \in \mathcal{F}^{*} \backslash \mathcal{U}_{b}^{*}$ for branching at the root node $\varphi$ of $T_{r}$ and continue branching 
on false clauses in $\mathcal{F}^{*}$ along clause $\mathcal{R}^{*}$ as if we were searching for a truth assignment for $\varphi^{*}$. Theorem 1 thus follows inductively also in the general case.

\section{References}

[1] E. Dantsin, A. Goerdt, E.A. Hirsch, R. Kannan, J. Kleinberg, C. Papadimitriou, O. Raghavan, U. Schoening [2002]: A deterministic $(2-2 /(k+$ 1) $)^{n}$ algorithm for $k$-SAT based on local search. In: Theoretical Computer Science 289 (2002), 69-83. Elsevier Science B.V.

[2] T. Hofmeister, U. Schoening, R. Schuler, O. Watanabe [2002]: A Probabilistic 3-SAT Algorithm Further Improved. In: H. Alt, A. Ferreira (Eds.): STACS 2002, LNCS 2285, 192-202. Springer-Verlag Berlin Heidelberg.

[3] U. Schoening [2002]: A Probabilistic Algorithm for $k$-SAT Based on Limited Local Search and Restart. In: Algorithmica 32 (2002), 615-623. Springer-Verlag New York Inc. 